\title{
El momento de las cadenas regionales de valor: la integración comercial en la Península Ibérica*
}

\author{
The Time of Regional Value Chains: \\ Trade Integration in the Iberian Peninsula
}

\author{
Carmen Díaz-Mora \\ Rosario Gandoy Juste \\ Belén González-Díaz \\ Universidad de Castilla-La Mancha
}

\begin{abstract}
Resumen
Desde la Gran Recesión, asistimos a un freno en el avance de las cadenas globales de valor $(C G V)$. Los factores que guiaron la globalización de estas cadenas desde mediados de los noventa han dejado de operar en dicha dirección, contribuyendo a una reconfiguración en la que se intensifica su dimensión regional. El objetivo del presente trabajo es investigar cómo la Península Ibérica ha participado de las tendencias de globalización y regionalización de las cadenas transfronterizas de producción en las que está integrada. Para ello, se analiza la participación de la Península Ibérica en las CGV, su sesgo regional y la integración bilateral de España y Portugal, que determina la existencia de un polo ibérico en el seno de las cadenas de producción.
\end{abstract}

Palabras clave: cadenas globales de valor, cadenas regionales de valor, Península Ibérica.

Clasificación JEL: F10, F14.

\begin{abstract}
Since the Great Recession, we observe a slowdown in the advance of Global Value Chains $(G V C)$. The factors that guided the globalization of those chains since the mid-1990s have ceased to operate in that direction, contributing to a reconfiguration of them with a tendency towards regionalization. The objective of this work is to investigate how the Iberian Peninsula has participated in these trends of globalization and regionalization of the cross-border production chains in which it is integrated. In this context, the present work analyses the participation of the Iberian Peninsula in the GVC, its regional bias as well as the bilateral integration of Spain and Portugal, which allow for the existence of an Iberian block within these production chains.
\end{abstract}

Keywords: global value chains, regional value chains, Iberian Peninsula.

JEL Classification: F10, F14.

* La segunda parte de este trabajo se apoya en un estudio previo, más amplio, realizado por las autoras con el título de «La periferia ibérica en las cadenas globales de valor» y presentado en el II Foro hispano portugués de internacionalización, celebrado el 26 de marzo de 2019, financiado y organizado por la Cátedra Global Nebrija Santander en internacionalización de empresas. 


\section{Introducción}

Desde mediados de los noventa, las cadenas globales de valor (CGV) se han configurado como uno de los rasgos más destacados de la globalización económica. Los avances en la liberalización comercial y los progresos técnicos en las comunicaciones y el transporte propiciaron la fragmentación internacional de los procesos productivos, reduciendo la incidencia de la distancia física en la localización de las diversas etapas que constituyen el proceso de fabricación. De acuerdo con su dotación factorial y ventajas comparativas, las empresas de las economías avanzadas desplazaron las fases de sus procesos productivos más intensivas en trabajo hacia aquellos emplazamientos con abundante mano de obra y menores costes laborales. Se desarrollan así redes de producción cada vez más globales que integran empresas de diversos y lejanos países (Los et al., 2015). Progresivamente, como resultado de la internacionalización de la producción, el centro de gravedad de la economía mundial se fue desplazando hacia el Este, con una creciente presencia de las economías emergentes y muy particularmente de China, cuya inserción en las CGV, especialmente en la producción de manufacturas, propició que a la altura de 2009 se convirtiese en el mayor exportador mundial de bienes, una posición que ha mantenido desde entonces.

Esta tendencia a la globalización de la producción, sin embargo, ha sido compatible con la permanencia de cadenas de valor organizadas en el ámbito regional. Es más, son estas las que tienen un papel predominante en el comercio mundial, pudiendo distinguirse en la actualidad tres grandes cadenas regionales que se organizan en torno al país que adopta una posición central en la red: «Factoría Norteamérica» constituida por EEUU, México y Canadá; «Factoría Asia», en torno a China, y «Factoría Europa», liderada por Alemania (Baldwin y López, 2015).

La Gran Recesión iniciada en 2008 supuso una ruptura en la senda de expansión de las CGV, que desde 2011 suspenden su avance. Diferentes autores han tratado de buscar explicaciones a este detenimiento, al que se responsabiliza del lento crecimiento del comercio mundial observado desde la crisis financiera global ${ }^{1}$. Constantinescu et al. (2015) argumentan que el shock tecnológico que impulsó el desarrollo de las CGV en los noventa ya ha sido absorbido y, en consecuencia, los procesos de fragmentación internacional de la producción se han ralentizado. Otro factor relevante a tener en cuenta son los cambios recientes en la dinámica de la participación china en CGV. Kee y Tang (2016) constatan un importante aumento del contenido doméstico en las exportaciones chinas de procesamiento que sugiere un proceso de sustitución de inputs importados por producción nacional. La menor demanda china de inputs intermedios importados, consecuencia de su upgrading en las cadenas de valor, ha tenido un efecto contractivo sobre el comercio mundial y las CGV. Por otra parte, como destacan Gaulier et al. (2015), la integración de China en

\footnotetext{
${ }^{1}$ Aunque la mayor parte de la literatura observa el detenimiento de las CGV, Gaulier et al. (2020), utilizando para su medición el comercio de partes y componentes y descontando la influencia de los precios y el ciclo económico, encuentran que las CGV mantienen su progresión tras la crisis financiera.
} 
CGV provocó cambios en la especialización comercial de acuerdo con las ventajas comparativas de los países y, dado el elevado volumen de sus exportaciones, ello ejerció una notable presión a la baja sobre los precios mundiales de los productos en los que China se especializaba, alentando, aún más, los procesos de fragmentación internacional. La reciente reorientación del modelo de crecimiento chino hacia un modelo más volcado en la demanda interna ha disminuido la presión competitiva en los mercados mundiales y, previsiblemente, sobre la internacionalización de los procesos productivos.

Adicionalmente, tras la crisis financiera, algunos de los principales factores que impulsaron el dinamismo de las CGV, como las ventajas salariales de las economías emergentes o los avances en la liberalización comercial y los desarrollos tecnológicos, han reducido o incluso cambiado el signo de su influencia sobre la internacionalización de la producción. Asimismo, algunos de los riesgos latentes asociados a las CGV se han materializado.

Por todo ello, recientemente se han acrecentado las voces que auguran el fin de la hiperglobalización y un proceso de desregulación y retroceso de las CGV que se manifestaría en el retorno de las actividades productivas anteriormente desplazadas por las empresas de las economías avanzadas (reshoring o backshoring) (Marin, 2020; Comisión Europea, 2019). Ahondando en esta cuestión, las estimaciones de la OCDE señalan que la digitalización y los avances técnicos serán el principal impulsor del cambio, provocando un acortamiento de las CGV y una reorientación de la actividad hacia los países miembros (De Backer y Flaig, 2017).

Sin embargo, en los últimos años se percibe también una tendencia a favor de la regionalización de las cadenas de valor, particularmente, en Europa. McKinsey (2019) aprecia, desde 2013, un incremento de la participación de los flujos comerciales intrarregionales en el total del comercio, especialmente en la Unión Europea (UE-28) y la región de Asia-Pacífico. El aumento es particularmente pronunciado en las CGV más intensivas en conocimiento (informática, electrónica, automóviles y otro material de transporte y química). Stollinger et al. (2018), utilizando el valor añadido doméstico reexportado como indicador de la participación en CGV, observan cómo, en 2012-13, se interrumpe la senda descendente que desde 2003 venían mostrando los intercambios intracomunitarios vinculados a cadenas de valor, para iniciarse una tibia recuperación de su presencia relativa. Piatanesi y Arauzo-Carod (2019) sugieren un crecimiento significativo en los próximos años del nearshoring, es decir, de la relocalización de actividades industriales desde los países en los que se deslocalizaron con anterioridad a países cercanos al lugar de origen de la actividad. Esta estrategia parece especialmente adecuada puesto que proporciona una respuesta a la disyuntiva entre mantener la actividad en el exterior o retornarla a casa, combinando las ventajas de la deslocalización (menores costes...) con la proximidad cultural y geográfica a la empresa matriz.

En este contexto de impulso a la regionalización de las redes transnacionales producción, este trabajo tiene por objeto analizar la participación y posición que ocupa la Península Ibérica en las cadenas regionales europeas y, más concretamente, inda- 
gar en el grado de integración comercial e intensidad de los intercambios bilaterales de España y Portugal en el seno de las cadenas de valor europeas. El análisis se basa en los indicadores desarrollados a partir de los flujos de comercio en valor añadido para el periodo 2000-2014 que se deducen de las tablas input-output internacionales $(W I O D)^{2}$, siguiendo el marco conceptual expuesto en Koopman et al. (2010 y 2014).

El trabajo se estructura en los siguientes apartados. Tras esta introducción, en la sección 2 se examinan las razones que apuntan hacia una reorientación de las cadenas de valor desde el ámbito global hacia el regional. El apartado tercero analiza, en primer lugar, la evolución de la participación en CGV de España y Portugal, de forma comparada con otros países de la UE y desglosando la dimensión sectorial; y, en segundo lugar, explora las relaciones comerciales bilaterales de ambos países en el marco de las CGV. En el apartado 4 se hace un análisis sectorial de tal integración bilateral, prestando atención especial a un sector primordial para nuestra economía, vehículos de motor. El trabajo concluye con unas consideraciones finales.

\section{La regionalización de las cadenas de valor}

La tendencia hacia la regionalización de las CGV puede explicarse a partir del marco teórico desarrollado en Baldwin (2011). En este esquema, la expansión de las CGV es el resultado de la confrontación entre las fuerzas que favorecen la deslocalización de la actividad (el ahorro de costes, básicamente, laborales, pero también otros, como los medioambientales) y las que impulsan su concentración geográfica, por ejemplo, las economías de aglomeración, los costes del comercio y los riesgos asociados a la fragmentación. La decisión y localización óptima de las tareas dependerá del balance entre el ahorro de costes en el desempeño de las tareas deslocalizadas y el conjunto de costes que implica la fragmentación del proceso productivo (comunicación, coordinación, gestión, control de calidad...). Como estos elementos varían en el tiempo, la configuración y desarrollo de las CGV depende de las transformaciones que tengan lugar en el entorno general. Es decir, las CGV son dinámicas, si bien, la intensidad de los costes hundidos asociados al establecimiento de la red limita su adaptación a las condiciones del mercado y la producción, de tal modo que, como la evidencia empírica ha mostrado, los flujos comerciales vinculados a CGV tienden a ser más estables (Diaz Mora et al., 2018).

Durante la década de los noventa y hasta bien entrado el siglo xxi predominaron las fuerzas a favor de la deslocalización de actividades. Los avances en la liberalización comercial, especialmente en las economías emergentes donde la protección comercial era más intensa, la proliferación de acuerdos comerciales, la incorporación de economías emergentes al mercado internacional, principalmente China, pero también del bloque de países del Este europeo tras la caída del muro de Berlín, las considerables

${ }^{2}$ La base de datos WIOD en su edición de 2016 incluye la información correspondiente a 43 países: los 28 de la Unión Europea más otros quince países relevantes en la producción y comercio mundial (Australia, Brasil, Canadá, China, India, Indonesia, Japón, México, Noruega, Rusia, Suráfrica, Corea, Suiza, Taiwán, Turquía y EEUU). Entre todos suponen más del $85 \%$ de la producción mundial (Timmer et al., 2016). 
diferencias en costes laborales entre las economías avanzadas y las naciones en desarrollo y la reducción en los costes de transporte y comunicaciones alentaron extraordinariamente los procesos de fragmentación internacional de la producción.

La Gran Recesión altera profundamente el panorama descrito. Los factores que guiaron la expansión de las CGV dejan de operar en dicha dirección. Asimismo, algunos de los riesgos latentes en la dispersión geográfica de los procesos productivos se hacen patentes, generando una notable incertidumbre sobre la estrategia. En este nuevo entorno, el balance de fuerzas que había alentado el desarrollo de las CGV se desequilibra y las CGV tienden a reconfigurarse aumentando el atractivo de localizaciones más cercanas geográfica y culturalmente: intensificándose las cadenas regionales de valor.

En el Gráfico 1 se presenta la evolución en la UE-28 del comercio de manufacturas vinculado a cadenas de valor, utilizando como indicador el porcentaje de valor añadido foráneo que se incorpora en las exportaciones (lo que en la literatura se denomina participación backward). De acuerdo con la procedencia de los inputs importados, se distingue entre la participación en cadenas regionales (CRV), si los flujos proceden de otros países del área, o en CGV, cuando los suministradores son terceros países. Puede observarse cómo, desde comienzos de siglo, las redes de producción se han ido haciendo más globales, hasta superar en importancia a las de dimensión regional. Esta tendencia se quiebra en 2012, iniciándose un progresivo retroceso de las CGV. En cambio, las CRV se muestran más estables, manteniendo una senda creciente ininterrumpida que les ha permitido recuperar su predominio.

\section{GRÁFICO 1}

VALOR AÑADIDO FORÁNEO DE LA UE-28 EN LAS EXPORTACIONES

DE MANUFACTURAS, 2000-2014

(En \% de las exportaciones de manufacturas)

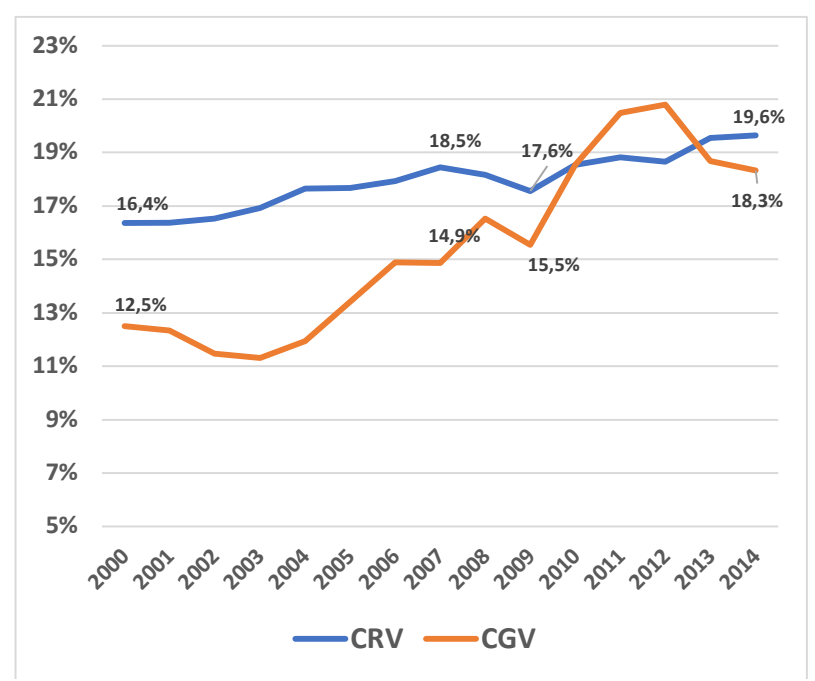

FUENTE: Elaboración propia a partir de WIOD (edición 2016). 
El resto del epígrafe se dedica a analizar los principales cambios en el entorno económico y geopolítico que inclinan la balanza hacia una reconfiguración de las CGV en las economías avanzadas, con localizaciones de las etapas más próximas a las sedes centrales de las empresas, es decir, que refuerzan las cadenas regionales de valor.

\section{a) Cambios en los costes relativos de producción y comercio}

El primer aspecto a considerar es la reducción en el diferencial de costes entre economías avanzadas y emergentes. Como consecuencia del crecimiento económico en China y otras economías en desarrollo, la brecha salarial se ha reducido, disminuyendo el atractivo de la deslocalización hacia las economías de bajo coste ${ }^{3}$. Esta tendencia se ha visto reforzada por avances tecnológicos como la robotización que, al restringir la intensidad en trabajo de las producciones, especialmente de las tareas más rutinarias que son las más susceptibles de deslocalización, reduce el interés en la deslocalización para aprovechar las ventajas laborales de los países emergentes. A este respecto, McKinsey (2019) destaca la caída en Europa y Asia, entre 2007 y 2017, de las importaciones basadas en ahorro de costes laborales. El trabajo de Strange y Zucchella (2017) revela, además, el importante potencial de la Industria 4.0 (internet de las cosas, big data...) como elemento disruptor en la localización y organización de las actividades dentro de las CGV. No obstante, aunque la idea más generalizada es que la robotización y las nuevas tecnologías digitales favorecerán el retorno a las economías avanzadas de actividades anteriormente deslocalizadas, algunos trabajos recientes apuntan que las nuevas tecnologías de producción (automatización, impresoras 3D, plataformas digitales, blockchain ...) pueden alentar la participación en CGV y el comercio, al reducir los costes del comercio e impulsar mejoras competitivas (Antras, 2020; Artuc et al., 2018).

Progresivamente, el ahorro en costes laborales ha ido cediendo paso entre los determinantes de la localización en los procesos de fragmentación internacional a otro tipo de factores, entre los que se cuenta el acceso a capital humano, la calidad de las infraestructuras y la proximidad a los mercados de consumo. Un proceso que mejora la posición de economías, con ciertas ventajas de costes laborales, cercanas a la ubicación de las empresas que lideran las cadenas. Un buen ejemplo es la estrategia de sustitución de proveedores asiáticos por italianos llevada a cabo por la empresa sueca Ikea. La tradicional especialización italiana en muebles de diseño y alta gama y el equilibrio entre innovación, calidad y coste de la mano de obra constituyeron un elemento primordial en la toma de decisión sobre la ubicación de los nuevos eslabones de la cadena productiva.

${ }^{3}$ A ello se añade la moderación salarial en las economías desarrolladas más afectadas por la crisis financiera. 
A esta evolución de los costes laborales relativos, se añade el incremento en los costes del comercio, especialmente en los años más recientes, cuando las tendencias proteccionistas se han manifestado con mayor contundencia. Aunque las tensiones comerciales se hacen particularmente evidentes a raíz de las medidas arancelarias sobre las importaciones chinas tomadas en 2018 por la Administración Trump, lo cierto es que, de una manera más sutil, a través del establecimiento de barreras no arancelarias, el proteccionismo ha experimentado un notable incremento desde la crisis financiera de 2008 (Gordo et al., 2020). En la actualidad, en un mundo globalizado, donde el $50 \%$ del comercio mundial de una manera u otra está vinculado a las CGV (World Bank, 2020), y los bienes intermedios cruzan diversas fronteras incrementando su valor añadido hasta alcanzar su destino final, los efectos del proteccionismo se magnifican, afectando al conjunto de economías que conforman la cadena de valor. Políticas industriales orientadas al mercado interno («Made in China 2025»; «America First»), exigencias de requerimiento local y otras medidas regulatorias que elevan los costes del comercio han tendido a frenar la expansión de las CGV, cuando no a revertirlas.

En esta escalada proteccionista, la distancia adquiere un renovado papel en las decisiones de localización de la actividad y en la reconfiguración de las cadenas de valor. Antrás y de Gortari (2020) desarrollan un modelo de equilibrio general en el que analizan la especialización dentro de las CGV en un mundo con barreras al comercio y encuentran que, cuando hay costes del comercio, la localización óptima de cada etapa depende no solo del coste marginal sino también de la proximidad geográfica a la etapa anterior y posterior. Su estimación de la importancia relativa de las cadenas regionales (NAFTA) respecto a las globales en EEUU adopta una forma de U invertida; de modo que, partiendo de la situación de equilibrio, incrementos en los costes del comercio provocan que las CGV reduzcan su importancia relativa a favor de las cadenas regionales. Un resultado acorde con la evidencia que encuentran Sturgeon y Van Biesebroeck (2009) para la industria del automóvil. Políticas proteccionistas e intervenciones públicas de distinto tipo a favor de la producción y el empleo en el territorio nacional explican la fortaleza y persistencia de las redes regionales de automoción, así como la localización de las actividades de ensamblaje e inputs especializados en países con elevados costes laborales. Tan solo en las partes y componentes más estandarizados se observa un desplazamiento de la actividad hacia economías distantes, como las asiáticas, para aprovechar las ventajas de costes y economías de escala.

\section{b) Riesgos inherentes a las $C G V$}

La existencia de riesgos inherentes a las CGV es uno de los factores que más pesan en contra de la fragmentación internacional. Riesgos que van desde la dificultad de gestionar con éxito una actividad dispersa en la que colaboran empresas de países con culturas y ámbitos institucionales muy distintos, a la posibilidad de interrupción 
de la cadena de producción con sus consiguientes repercusiones en los resultados empresariales.

El terremoto de Japón, en 2011, supuso la primera gran llamada de atención de que los riesgos de interrupción de la cadena podían materializarse. Al destrozar las plantas de algunos de los mayores fabricantes de componentes de electrónica y automoción se paralizó la producción de los posteriores eslabones de la cadena de valor, expandiéndose sus consecuencias económicas a diferentes países. Fabricantes de automóviles en Detroit tuvieron que cerrar temporalmente por falta de suministros.

La actual pandemia COVID-19 ha vuelto a poner de manifiesto los riesgos que implica la dependencia productiva del exterior. Su origen en China, y concretamente en Wuhan, capital de una de las provincias más industrializadas, de donde proceden una importante parte de suministros de las industrias más intensivas en tecnología ${ }^{4}$, trasladó, a través de las CGV, los efectos de la paralización de la actividad en China por todo el mundo. La escasez de productos nacionales para hacer frente a la pandemia ha acentuado las voces que, en las economías avanzadas, claman por la necesidad de reducir la dependencia externa, el retroceso de las CGV y el retorno de la actividad productiva. Sin embargo, como mencionan Baldwin y Tomiura (2020), las CGV se han desarrollado con el objeto de mejorar la eficiencia productiva y su reversión tiene el efecto contrario sobre la productividad, competitividad y crecimiento. La propuesta de estos autores para hacer frente a la excesiva importancia que ha cobrado China en los suministros no es, por tanto, el desmantelamiento de las CGV, sino su reconfiguración, con una diversificación en el origen de los suministros que reduzca los riesgos.

En esta diversificación de proveedores, la proximidad geográfica y cultural debería ser un factor determinante, particularmente en lo que respecta a los inputs específicos, aquellos que son imprescindibles para el desarrollo de determinadas actividades. Los lazos establecidos entre economías cercanas y afines, especialmente cuando existe un marco institucional común que puede venir determinado por acuerdos comerciales de integración, son un plus de garantía para impedir y, en su caso, resolver conflictos en los suministros ${ }^{5}$.

Especial preocupación genera en las economías avanzadas la dependencia de importaciones de inputs que incorporan las tecnologías más avanzadas, particularmente las procedentes de China. La inquietud en EEUU por el dominio tecnológico chino se evidencia en la utilización del argumento de «seguridad nacional» como justificación de las medidas proteccionistas adoptadas: el establecimiento de aranceles sobre importaciones tecnológicas de China, el bloqueo a Huawei o las limitaciones

${ }^{4}$ Según The Economist (2020), una cuarta parte de los cables y dispositivos de fibra óptica se producen en esta provincia. Destaca, asimismo, su relevancia como proveedor mundial de partes y componentes de automoción.

${ }^{5}$ Los conflictos en el abastecimiento desde el exterior se han evidenciado en la reciente crisis sanitaria cuando los países han tenido que competir en el mercado mundial por la adquisición de material sanitario. La paralización en Francia de un envío de mascarillas procedente de China y dirigido a España e Italia, solo encontró solución tras la apelación al carácter de socios/amigos de los países de destino. 
al acceso de tecnología americana. De hecho, la reciente política comercial estadounidense es, en gran medida, la respuesta a las políticas industriales intervencionistas que ha venido aplicando China con el propósito de situarse entre los países líderes en sectores estratégicos de alta tecnología, reduciendo su dependencia tecnológica del exterior y escalando en las CGV en las que participa (estrategia «Made in China 2025»). En Europa, tiene su reflejo en la inclusión de la «soberanía tecnológica» entre las prioridades de la política industrial comunitaria. Si bien, en este caso, las actuaciones van en la dirección de reforzar la cooperación europea en innovación y desarrollo tecnológico, apoyando el desarrollo de cadenas de valor, fundamentalmente a nivel europeo, claves para la competitividad. Un ejemplo es la European Battery Alliance, creada en 2017 para desarrollar una cadena de valor europea en torno a celdas de baterías que asegure la soberanía tecnológica y la competitividad de la industria europea de automoción (Comisión Europea, 2019).

\section{c) La crisis del multilateralismo}

Los acuerdos multilaterales son importantes para las CGV dado que ayudan a definir las reglas del juego estableciendo normas sobre distintos aspectos que afectan a los intercambios comerciales; facilitando, en consecuencia, la viabilidad de procesos productivos dispersos. Por ello, la crisis del multilateralismo que caracteriza las relaciones comerciales en los años más recientes afecta negativamente a las CGV. La incapacidad de la Organización Mundial del Comercio (OMC) para hacer frente a los retos que plantea el nuevo orden económico internacional y solventar las dificultades asociadas al mecanismo de resolución de diferencias ha generado una notable incertidumbre, alentando la aparición de las tensiones comerciales ya comentadas y un viraje hacia el bilateralismo, con efectos adversos sobre las $\mathrm{CGV}$, que requieren de un marco común y estable.

En este contexto, las CRV se enfrentan a una menor incertidumbre, puesto que para garantizar su viabilidad e impulsar las ganancias de bienestar a ellas asociadas es suficiente la dimensión regional de los acuerdos comerciales (Los et al., 2015). Es más, las estimaciones realizadas muestran el impacto positivo que los acuerdos comerciales regionales han tenido sobre la participación en cadenas transnacionales de valor, especialmente en la Unión Europea y ASEAN. Además, el impacto es mayor cuanto más profundo es el acuerdo regional (Johnson y Noguera, 2017) ${ }^{6}$. Ahondando en esta cuestión, Boffa et al. (2018) muestran un efecto especialmente favorable sobre la participación en cadenas de valor de la implementación conjunta de medidas que afectan al comercio y la inversión. Dado que las decisiones empresariales acerca de la localización de las etapas productivas vienen determinadas por ambas

\footnotetext{
${ }^{6}$ Los acuerdos comerciales profundos incorporan materias adicionales a las que tradicionalmente considera la OMC, cubriendo aspectos normativos en inversiones, compras públicas, política de competencia, propiedad intelectual, mercado de trabajo, etc.
} 
cuestiones, la existencia de regímenes comunes supone un atractivo adicional para el territorio. Así, las regiones que se benefician de acuerdos comerciales profundos, como es el caso de la Unión Europea, están mejor posicionadas frente a la creciente incertidumbre y sus cadenas regionales de valor podrían salir reforzadas.

\section{España y Portugal en las CGV}

Una vez puesta de manifiesto la tendencia hacia la regionalización de las redes transnacionales de producción y explorado los cambios en el entorno económico y geopolítico que lo están propiciando, nos centrarnos en un aspecto concreto de tal regionalización como es la existencia de un polo ibérico dentro de las cadenas europeas de producción. Para ello, en primer lugar, analizamos la evolución de la participación en CGV de España y Portugal para, en segundo lugar, explorar las relaciones comerciales bilaterales de ambos países en el seno de tales CGV.

\subsection{La participación de España y Portugal en las CGV}

Para analizar la participación de Portugal y España en las CGV adoptamos una perspectiva dinámica y comparada con las economías centrales de la UE, Alemania, Francia e Italia, y con los llamados países Visegrad, Polonia, República Checa, Hungría y Eslovaquia, grupo de países que, tras su integración al proyecto europeo, se han incorporado de forma más intensa a las cadenas de valor europeas.

Utilizando como indicador el porcentaje de valor añadido foráneo que se incorpora en las exportaciones, con carácter general, se observa un aumento del contenido importado entre 2000 y 2011, una vez salvada la caída puntual de 2009 asociada al colapso del comercio mundial en dicho año (Gráfico 2). Sin embargo, a partir de 2011, el avance de las CGV se detiene en la Península Ibérica, e incluso se retrae en el resto de las economías, salvo la República Checa, coincidiendo con lo que se viene observando para el conjunto de la economía mundial. Tanto el nivel como la evolución de esta participación en CGV, nos sitúa, junto a Polonia, en una posición intermedia en el ámbito europeo: entre las economías del Este más inmersas en CGV y las economías de mayor peso económico y tamaño, con un valor añadido importado que supone en torno al $30 \%$ de las exportaciones. Destaca la similitud que muestran los indicadores de España y Portugal a partir de 2010, porque hasta entonces la dependencia importadora de Portugal había estado claramente por encima de la española, como corresponde a su menor dimensión. 


\section{GRÁFICO 2}

\section{VALOR AÑADIDO FORÁNEO EN EL TOTAL DE EXPORTACIONES, 2000-2014}

\section{(En \%)}

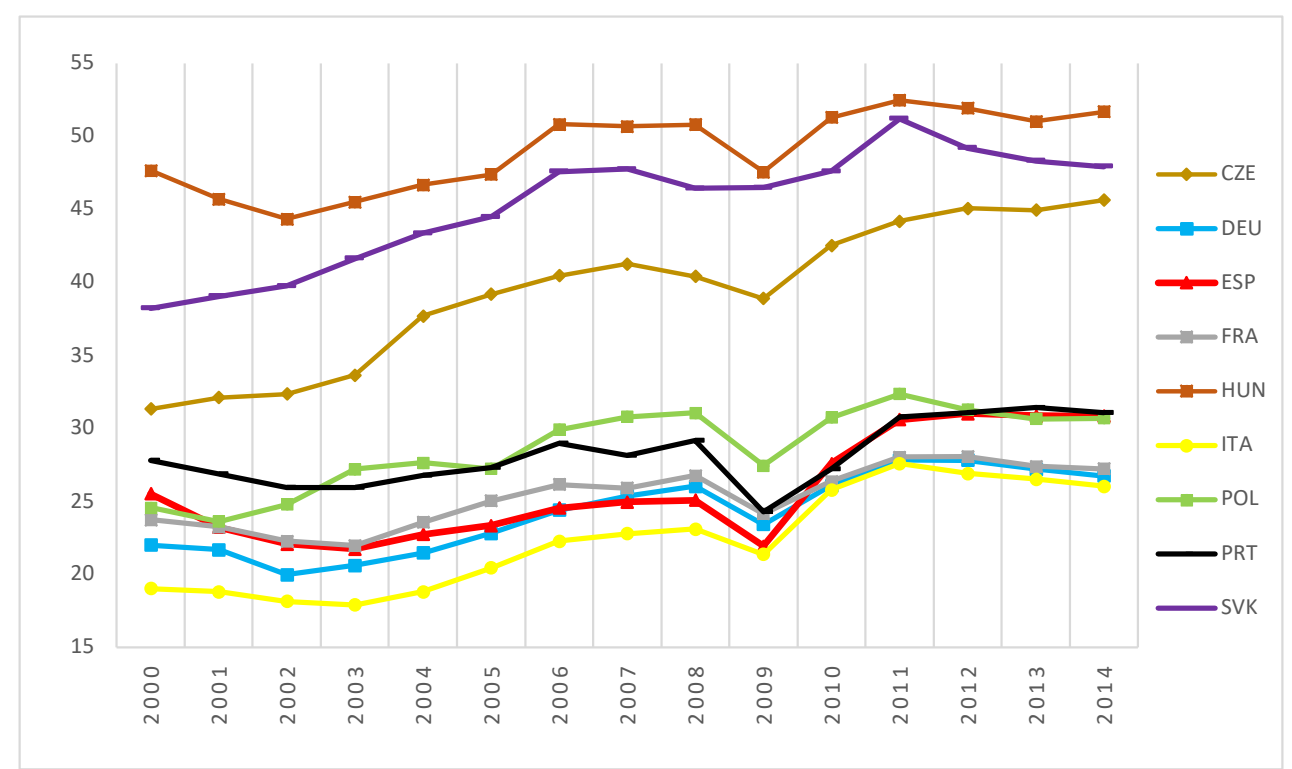

NOTA: CZE (República Checa), DEU (Alemania), ESP (España), FRA (Francia), HUN (Hungría), ITA (Italia), POL (Polonia), PRT (Portugal), SVK (Eslovaquia).

FUENTE: Elaboración propia a partir de WIOD (edición 2016).

Cuando analizamos la participación de ambas economías ibéricas en CGV por sectores económicos, encontramos que es particularmente activa en las ramas manufactureras (Gráfico 3). Para España, los porcentajes más elevados de contenido importado, tras ascensos importantes en el periodo analizado, se alcanzan en coque y refino de petróleo (tres cuartas partes), fruto de nuestra marcada dependencia energética del exterior, vehículos de motor (la mitad) y metálicas básicas y productos químicos (rondando el $40 \%$ ). Por el contrario, y resultado de un retroceso o avances muy limitados, el contenido importado es inferior, por un lado, en producciones más vinculadas a recursos naturales como las agroalimentarias, extractivas o el sector maderero y, por otro, en los servicios. Para Portugal, la intensidad y ordenación sectorial de la participación en CGV es muy parecida. No obstante, pueden destacarse como rasgos diferenciales de Portugal un contenido importado superior en los bienes de capital (Maquinaria y bienes de equipo, equipos informáticos y material de transporte) y una menor dependencia importadora, es decir, mayor contenido nacional de sus exportaciones en textil y confección y productos farmacéuticos. 
GRAFICO 3

VALOR AÑADIDO FORÁNEO EN LAS EXPORTACIONES SECTORIALES, 2000 Y 2014

(En \%)

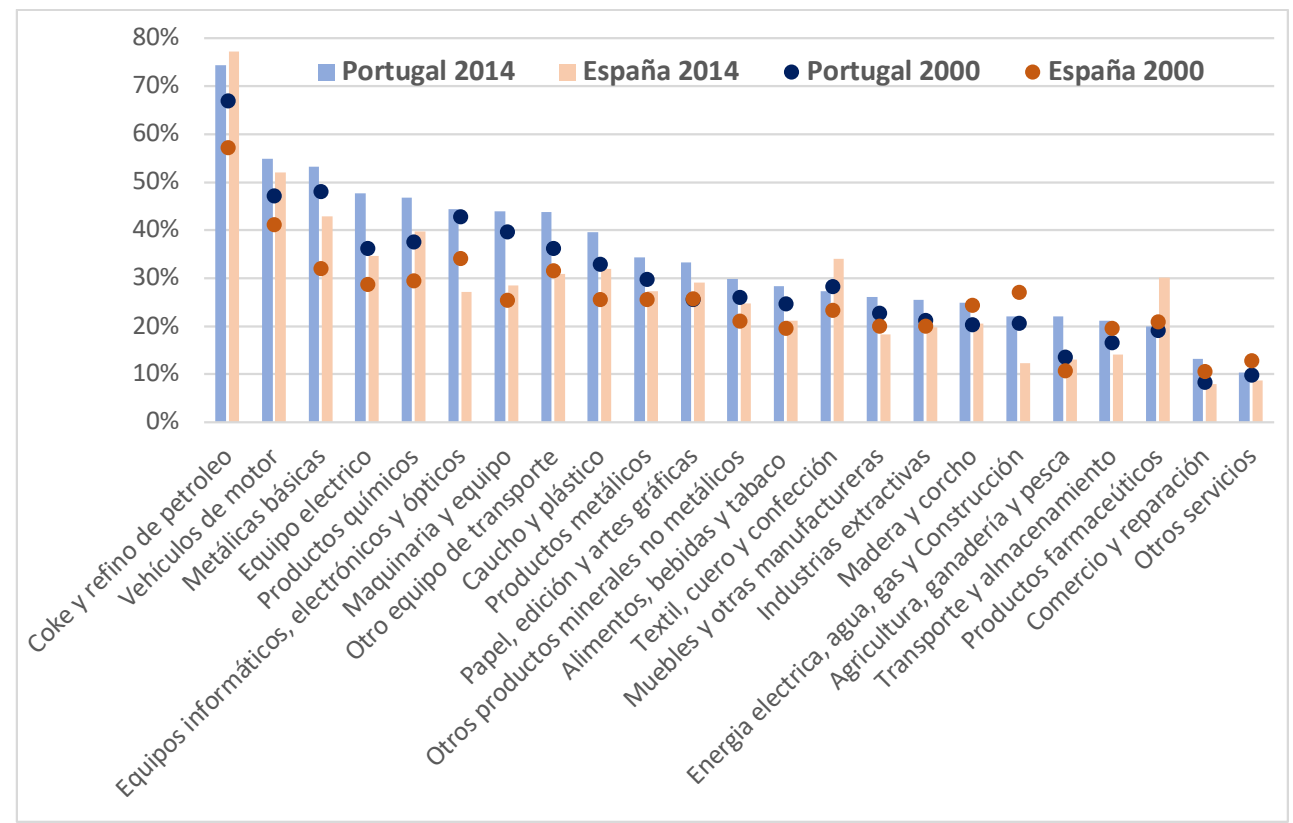

FUENTE: Elaboración propia a partir de WIOD (edición 2016).

\subsection{La integración bilateral en la Península Ibérica}

En este apartado exploramos las relaciones comerciales bilaterales entre España y Portugal en el marco de las CGV para determinar si su avance en ambos países ha supuesto una mayor integración comercial regional en el contexto europeo y más concretamente en la Península Ibérica. El origen geográfico de los inputs importados que España y Portugal incorporan a sus exportaciones permite aproximar la intensidad y evolución de la participación de ambos países en CGV y CRV (Cuadro 1, primeras dos columnas).

Lo primero que observamos es esa doble dimensión global y regional de las cadenas de producción transfronterizas en las que participan tanto España como Portugal, que se inclina ligeramente hacia la global en España y hacia la regional en Portugal. El componente global se ha comportado más dinámico en el conjunto del periodo en ambas economías. No obstante, su avance se ha frenado en los años más recientes (2013-2014), mientras que se mantiene el peso del componente regional.

Para el caso de España, son las tres grandes economías de la Unión Europea, Alemania, Francia e Italia (en ese orden), las que se sitúan a la cabeza del ranking 
de proveedores de inputs intermedios a nuestra economía. El protagonismo de Alemania es una expresión de su liderazgo en las CRV europeas derivado de su tamaño económico y, sobre todo, de su especialización en maquinaria y material de transporte, algunas de las manufacturas donde las CGV tienen una mayor presencia. Aunque también Portugal se encuentra entre el grupo de nuestros principales proveedores de inputs intermedios, su contribución (en torno al $2 \%$ de esos inputs foráneos y al $0,6 \%$ de las exportaciones españolas) es inferior a la de otras economías avanzadas y de mayor dimensión. Por su parte, España es el principal proveedor de inputs intermedios para Portugal (sumando el $18 \%$ de esos inputs foráneos incorporados en sus exportaciones y el 5,5\% de tales exportaciones lusas), indicando una arraigada integración de Portugal en CRV ibéricas, tal y como destacan otros autores (Amador y Stehrer, 2014) y explicando el predominio del componente regional. A España le siguen las grandes economías europeas, Alemania, Francia e Italia. Así, la acusada dependencia de inputs foráneos procedentes de las grandes economías europeas es un rasgo que también caracteriza a las exportaciones portuguesas. Por tanto, los datos analizados confirman que España y Portugal están perfectamente integradas en las CRV de dimensión europea.

CUADRO 1

PARTICIPACIÓN EN CGV DE ESPAÑA Y PORTUGAL (En \% sobre las exportaciones y cambio en puntos porcentuales)

\begin{tabular}{|c|c|c|c|c|c|c|c|c|}
\hline & \multicolumn{4}{|c|}{$\begin{array}{l}\text { Contenido importado } \\
\text { de las exportaciones }\end{array}$} & \multicolumn{4}{|c|}{$\begin{array}{l}\text { Valor añadido doméstico } \\
\text { exportado y reexportado por } \\
\text { el país de destino }\end{array}$} \\
\hline & \multicolumn{2}{|c|}{ España } & \multicolumn{2}{|c|}{ Portugal } & \multicolumn{2}{|c|}{ España } & \multicolumn{2}{|c|}{ Portugal } \\
\hline & 2014 & $\begin{array}{c}\text { Cambio } \\
\text { 00-14 }\end{array}$ & 2014 & $\begin{array}{c}\text { Cambio } \\
\text { 00-14 }\end{array}$ & 2014 & $\begin{array}{c}\text { Cambio } \\
\text { 00-14 }\end{array}$ & 2014 & $\begin{array}{c}\text { Cambio } \\
00-14\end{array}$ \\
\hline Total & 30,8 & 5,3 & 31,1 & 3,3 & 20,1 & 1,3 & 22,3 & 4,5 \\
\hline $\begin{array}{l}\text { Desglose por países } \\
\text { (origen/destino) } \\
\text { Alemania }\end{array}$ & 3,5 & $-0,1$ & 3,5 & $-0,2$ & 2,6 & 0,1 & 2,3 & 0,2 \\
\hline Francia & 3,2 & $-1,0$ & 2,1 & $-0,6$ & 2,3 & $-0,3$ & 1,8 & $-0,1$ \\
\hline Italia & 1,8 & $-0,3$ & 1,5 & $-0,4$ & 1,2 & 0,1 & 0,8 & 0,1 \\
\hline Reino Unido & 1,0 & $-1,2$ & 1,1 & $-0,9$ & 0,8 & $-0,5$ & 0,8 & $-0,3$ \\
\hline Portugal & 0,6 & 0,2 & - & - & 1,1 & 0,3 & - & - \\
\hline España & - & - & 5,5 & 0,5 & - & - & 3,2 & 0,6 \\
\hline EEUU & 1,4 & $-0,2$ & 1,2 & $-0,2$ & 0,4 & $-0,1$ & 0,4 & 0,0 \\
\hline China & 1,6 & 1,3 & 1,2 & 1,0 & 0,5 & 0,4 & 0,6 & 0,4 \\
\hline Países Visegrad & 0,9 & 0,6 & 0,6 & 0,2 & 1,0 & 0,5 & 0,8 & 0,5 \\
\hline CRV & 13,9 & $-1,6$ & 17,0 & $-1,7$ & 12,4 & 0,1 & 13,2 & 1,5 \\
\hline CGV & 16,9 & 6,9 & 14,1 & 4,9 & 7,7 & 1,2 & $\mathbf{9 , 1}$ & 3,0 \\
\hline
\end{tabular}

FUENTE: Elaboración propia a partir de WIOD (edición 2016). 
La evolución de la contribución relativa de los distintos países al valor añadido foráneo contenido en las exportaciones sugiere, sin embargo, una cierta reorientación en el origen de los inputs exteriores de la Península Ibérica. En ambos países, aunque con más intensidad en España, se registra una pérdida de peso de las economías más avanzadas (Alemania, Francia, Italia, Reino Unido y EE UU) a favor, sobre todo, de China, los países Visegrad y de otras economías emergentes como Brasil, Rusia o India. Es la expresión de la creciente integración de ambos países en la economía mundial y de cómo las redes en las que participan ambos países se han ido haciendo cada vez más globales, en un esfuerzo de las empresas por internacionalizar su actividad y proveerse de inputs exteriores de países de bajo coste con objeto de mejorar su capacidad competitiva. Sin embargo, esta diversificación geográfica de los proveedores de inputs importados no ha supuesto un retroceso de la integración comercial en la región ibérica. Al contrario, tanto España como Portugal han fortalecido su peso como suministradores de inputs intermedios entre sí ( 0,2 puntos porcentuales el primero y medio punto el segundo), lo que evidencia la solidez de las cadenas de producción ibéricas.

El análisis de las relaciones comerciales entre España y Portugal en el seno de las CGV efectuado hasta este momento se ha centrado en determinar la relevancia del socio ibérico en el contenido importado de las exportaciones de ambos países. Una perspectiva complementaria es contemplar la integración comercial de ambos países desde la óptica del productor de los inputs que se incorporan en las cadenas productivas en las que participan (participación forward). Es decir, valorar cuál es para España y Portugal la importancia relativa de su socio ibérico como destino de los inputs intermedios que exportan para su transformación y posterior incorporación en exportaciones a terceros países (Cuadro 1, dos últimas columnas).

Para España, de nuevo, Alemania, Francia e Italia son los destinos prioritarios de los inputs intermedios españoles para su transformación y posterior reexportación, si bien con una progresiva pérdida de relevancia a favor de nuevos mercados, especialmente China y las economías Visegrad. Ello pone de manifiesto nuevamente como convive en España la tendencia hacia la dimensión global, participando de la reorganización de las CGV hacia Asia, con la solidez de la dimensión regional, participando de la reorganización de las redes europeas hacia la Europa del Este y fortaleciéndose el polo ibérico. Así, Portugal es el cuarto destino en importancia siendo, por tanto, más destacada su posición como destino de las exportaciones intermedias españolas que como proveedor de inputs en las CGV en que ambos países participan. Además, su posición experimenta un significativo avance desde comienzos de siglo.

Por su parte, para la economía lusa, Alemania y Francia son los principales destinos del valor añadido portugués reexportado, si bien, en este caso, claramente superados por España. Y también Portugal participa de la reorganización de las CGV a tenor de la pérdida de la presencia relativa de los socios europeos más avanzados en favor de China y, en menor grado, de economías europeas emergentes como la República Checa, Polonia o Hungría, lo que permiten afianzar la ya predominante dimensión sectorial de las cadenas transfronterizas en las que participa. 
Se confirma, por tanto, la intensa integración comercial de los dos países y su estrecha colaboración en las CGV en las que ambos participan, tanto en lo que se refiere a la dependencia de sus exportaciones de inputs procedentes del socio ibérico como en su papel como proveedores de inputs intermedios que se incorporan en las exportaciones del socio periférico.

Adicionalmente, realizamos una comparación de ambas vías de participación en CGV, lo que nos permite aproximar la posición de los países en las CGV. Así, las economías que participan en mayor grado a través de la adquisición, transformación y montaje de inputs intermedios exteriores (participación backward) se situarán en las etapas más adelantadas de la CGV, más cerca del consumidor final, mientras que aquellas en las que predomina el suministro de inputs intermedios a otras economías (participación forward) se posicionarán en las etapas iniciales. Cuando hacemos este ejercicio para España y Portugal, extraemos dos conclusiones principales.

En primer lugar, la participación en CGV de España y Portugal a través del suministro de inputs intermedios para su transformación y exportación posterior es inferior a la que llevan a cabo mediante la transformación de inputs importados, lo que apunta que ambos países tienden a situarse en las etapas de fabricación más próximas al consumidor final. Además, ha tenido lugar un retroceso desde el inicio de la crisis que parece indicar que la participación española en CGV se ha ido orientando en mayor medida hacia la transformación de inputs importados, al contrario de lo que ocurre en Portugal. En segundo lugar, en lo que respecta a las relaciones bilaterales en el seno de las CGV con las economías centrales europeas, tanto en España como Portugal, predomina su participación como transformadoras de inputs intermedios destinados a la exportación. No obstante, en las relaciones bilaterales España-Portugal prevalece la provisión de inputs intermedios de España a Portugal desde donde, tras su elaboración y ensamblaje, se reexportan a otros países, colocándose, por tanto, Portugal en etapas más cercanas al consumidor final en las CGV en las que ambos participan.

\section{Análisis sectorial}

En este epígrafe, descendemos al análisis sectorial para investigar hasta qué punto la participación en CGV y la integración comercial entre ambas economías descrita en el apartado anterior difiere según sectores. En el Gráfico 4 representamos, para 2014, en las abscisas, el valor añadido procedente del socio ibérico contenido en las exportaciones de cada rama en porcentaje de las exportaciones sectoriales, que expresa la dependencia de la rama respecto al socio. En las ordenadas se recoge el peso del socio en el total de valor añadido foráneo incorporado en las exportaciones, que permite distinguir en que ramas la integración comercial bilateral es más intensa. Para ambos indicadores, dibujamos con una línea el porcentaje promedio de la economía, de manera que los sectores situados en el cuadrante superior derecho son aquellos con valor añadido foráneo procedente del socio ibérico por encima de la media nacional, desde ambas perspectivas, y que identificamos como los sectores que más activamente están inmersos en redes bilaterales. 


\section{GRAFICO 4}

\section{VALOR AÑADIDO FORÁNEO PROCEDENTE DEL SOCIO IBÉRICO EN LAS EXPORTACIONES SECTORIALES, 2014 \\ (En \%)}
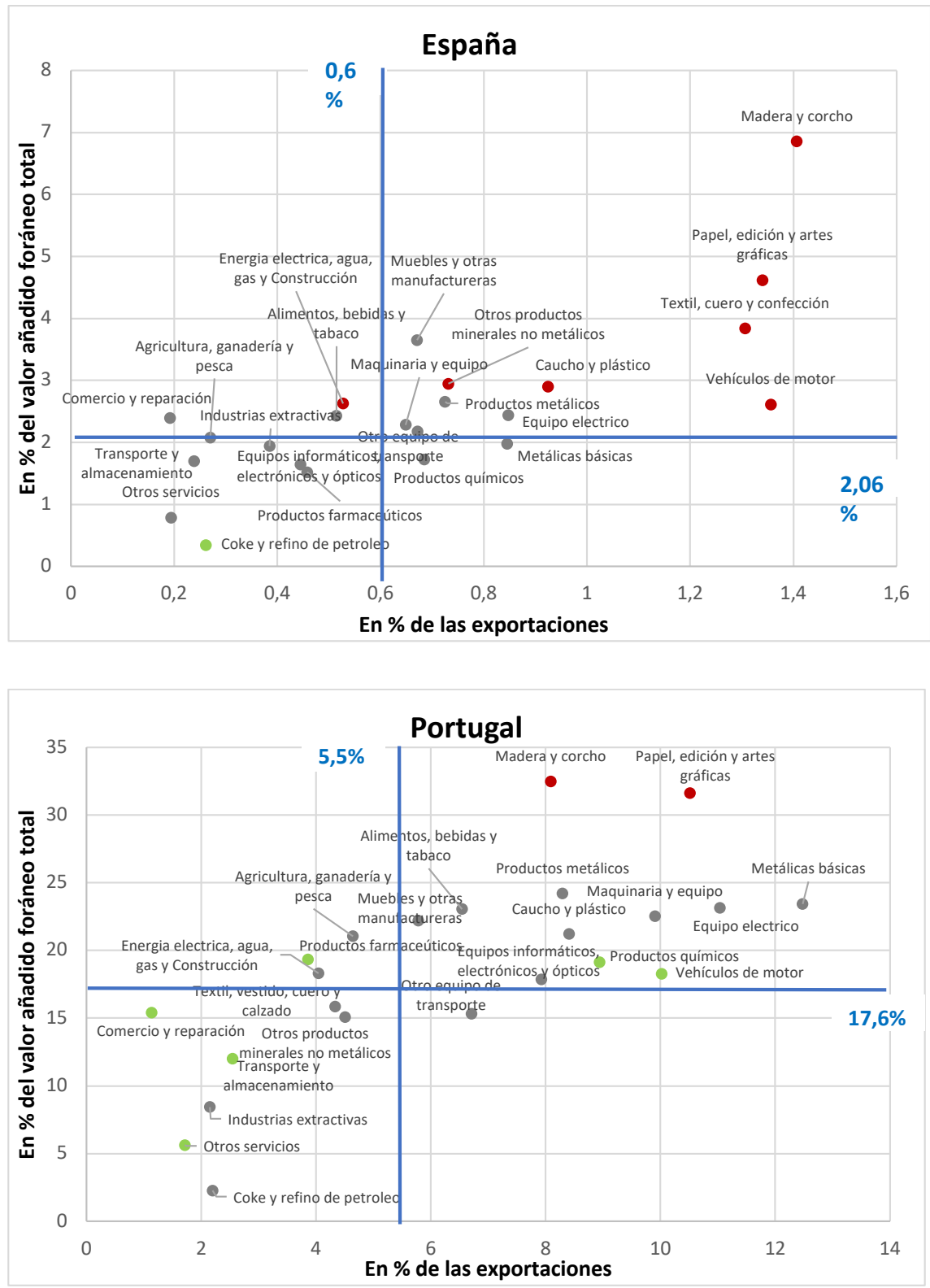

NOTA: Puntos rojos: aumentos, entre 2000 y 2014, del valor añadido foráneo procedentes del socio ibérico superiores a 1 punto porcentual para España y 5 para Portugal. Puntos grises: resto de aumentos. Puntos verdes: descensos de valor añadido foráneo en el periodo considerado.

FUENTE: Elaboración propia a partir de WIOD (edición 2016) 
Lo primero que observamos es que dicha integración bilateral es muy dispar según ramas de actividad en ambas economías, siendo particularmente baja en servicios y alta en algunas manufacturas. Entre ellas despuntan especialmente la industria de vehículos de motor y la de papel, edición y artes gráficas, en las que tiene lugar, para los dos países, una elevada dependencia de los suministros de su socio ibérico. Adicionalmente, la mayor dependencia en España de importaciones portuguesas se produce en industrias tradicionales como madera y corcho y textil y confección. Mientras que para Portugal es especialmente reseñable la dependencia de importaciones españolas para la producción de equipos eléctricos, metálicas básicas y maquinaria y equipos.

En cuanto a la evolución, el aumento de peso de Portugal como proveedor de inputs intermedios para las exportaciones de España detectado en el análisis agregado, tiene lugar en prácticamente todas las ramas (excepto coque y productos farmacéuticos), si bien lo hace de forma moderada; es algo más notorio en textil y confección y en madera y corcho, profundizando la integración en redes de producción ibérica en dichos sectores. Por su parte, la trayectoria que ha seguido el contenido importado procedente de España en las exportaciones portuguesas ha sido más dispar según sectores: descendente en cuatro ramas (tres de servicios y vehículos de motor), con apenas variación en otras siete ramas y con avances en el resto, siendo particularmente significativos en tres ramas, madera y corcho, papel y edición y agroalimentario. Son estos, por tanto, los sectores donde la colaboración entre ambas economías en el seno de las CGV se ha venido haciendo más estrecha.

A continuación, abordamos un análisis más detallado para uno de los sectores más involucrados en CGV en la esfera mundial y también, como se ha visto, en las cadenas de producción ibéricas. Se trata del sector de vehículos de motor, que es, además, un sector primordial en las exportaciones de ambos países.

Comenzamos analizando la posición, para este sector, que el socio ibérico ocupa en el ranking de principales proveedores de valor añadido foráneo contenido en las exportaciones de España y de Portugal en 2014 (Cuadro 2). Portugal se encuentra en octava posición como proveedor de valor añadido contenido en las exportaciones españolas, muy alejado de tres líderes europeos en automoción (Alemania, Francia e Italia), que son los que encabezan el ranking y que juntos suman más del $40 \%$ del valor añadido foráneo total. Estos datos constatan una marcada participación en cadenas de producción con el centro de Europa, que ha ido de la mano de inversión directa extranjera en España procedente de esos tres grandes países. No obstante, todos ellos han perdido peso como proveedores de inputs. Por el contrario, se ha fortalecido el polo ibérico al incrementarse el porcentaje de valor añadido foráneo procedente de Portugal. Es un resultado reseñable en tanto que es el único de los antiguos miembros que aumenta su presencia. Asimismo, se observa un fortalecimiento de las conexiones españolas hacia atrás con China, que se sitúa ya como el cuarto proveedor de inputs y nos dirige hacia una participación en redes de carácter más global. Y también aumentan las conexiones con el polo en automoción de Europa del Este, concretamente con Polonia que ha sido el segundo país, tras China, 


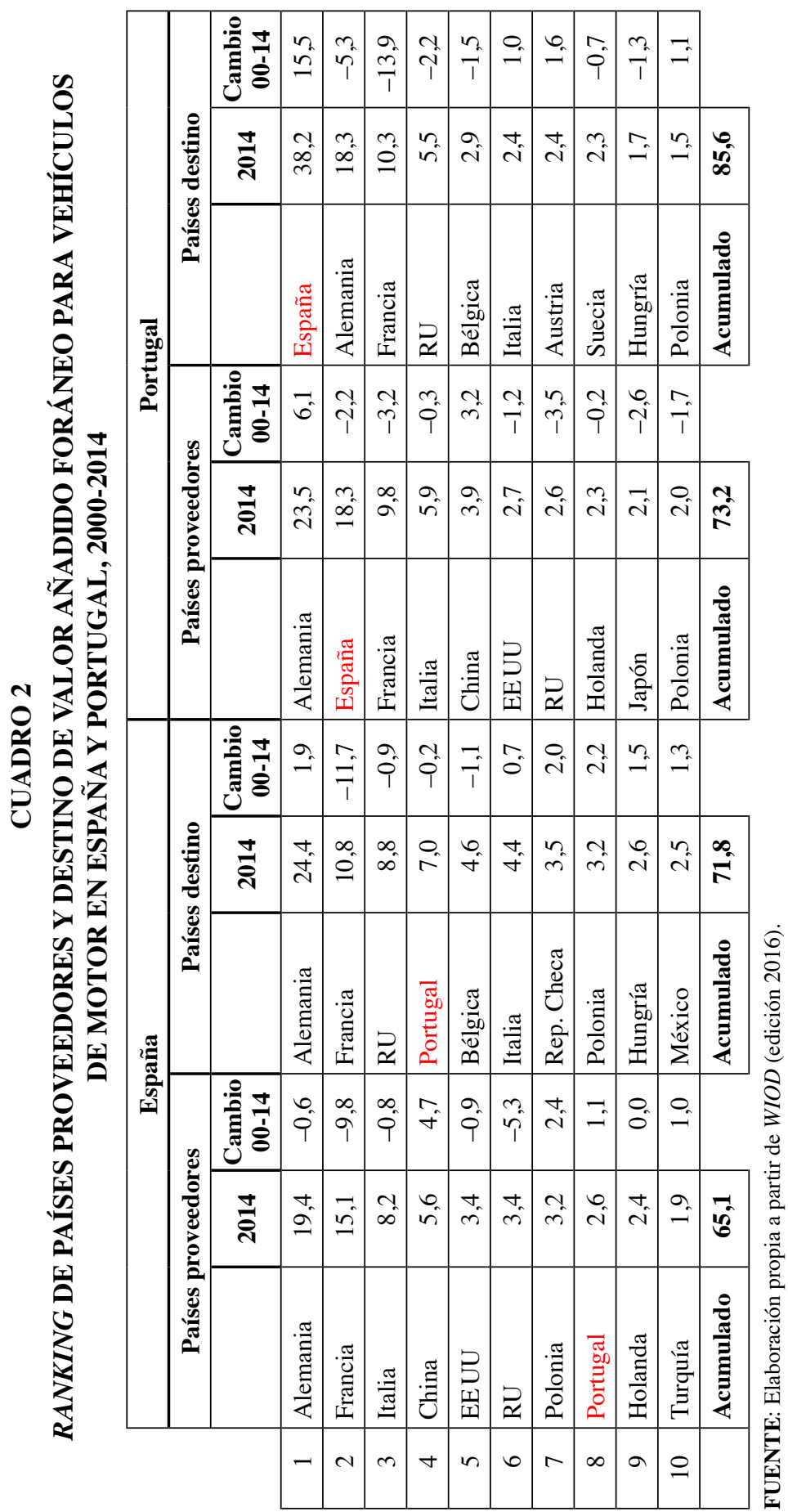


que más ha aumentado su peso en los inputs foráneos que incorpora el sector de vehículos de motor español. Se aprecia, por tanto, que España, en el periodo analizado, ha conseguido participar activamente del proceso de reconfiguración regional de las cadenas de producción en el contexto de la Europa ampliada y lo ha hecho manteniendo e incluso intensificando sus vínculos productivos con Portugal y Europa del Este. Estos rasgos enfatizan el carácter regional de la participación española en CGV en automoción.

Si ponemos el foco en Portugal, observamos el predominio de España como proveedor de inputs intermedios en vehículos de motor, con cerca del $20 \%$, ocupando el segundo lugar. Junto a España, se sitúan las economías centrales de la Unión Europea, Alemania, Francia e Italia, lo que denota una muy elevada integración de Portugal en CRV de dimensión europea, dentro de las cuales se aprecia claramente un polo ibérico. Respecto a la evolución en estos tres quinquenios, el polo ibérico se resiente levemente dado que España pierde protagonismo como proveedor de valor añadido a Portugal; también lo hacen otras economías europeas, a excepción de Alemania con la que Portugal ha aumentado muy sensiblemente su adquisición de inputs intermedios foráneos, desbancando a España como principal proveedor. De nuevo hay que resaltar la creciente importancia de China como origen de las compras intermedias que se incluyen en las exportaciones portuguesas, que apunta a una mayor integración lusa en CGV (Amador y Stehrer, 2014) y evidencia cambios en su arquitectura en este periodo.

Tal y como hemos hecho para el agregado de la economía, también para el sector concreto de automoción podemos analizar los vínculos forward (además de los vínculos backward), esto es, atendiendo a la importancia relativa del socio ibérico como destino (en vez de como origen) de los inputs intermedios que se incorporan a las exportaciones de terceros países, obteniendo conclusiones similares. Comenzando por España, de su valor añadido doméstico contenido en las exportaciones de otros países, los cuatro principales destinatarios son las tres mayores economías europeas, Francia, Alemania y Reino Unido, a las que se suma Portugal en el cuarto puesto. Aquí el carácter regional de la participación española en CGV es más evidente, pues los nueve principales destinos son economías europeas, concentrando el $70 \%$ de inputs foráneos incorporados en nuestras exportaciones sectoriales. Atendiendo a la evolución, se observa cierto fortalecimiento de la participación forward en CGV con economías emergentes, sobre todo de Europa del Este como Polonia o República Checa, que solo se produce a expensas de Francia, Reino Unido y Bélgica pues Portugal mantiene su peso y Alemania incluso lo aumenta. Como en los vínculos backward, la dimensión regional de las cadenas productivas en automoción en las que España participa se mantiene en lo que se refiere al polo ibérico y se amplía a Europa del Este.

En cuanto al valor añadido generado en Portugal que se incorpora en las exportaciones de terceros países, el liderazgo de España es rotundo (con el $40 \%$ del total), por delante de las grandes economías europeas. Además, ha ido ganando peso de forma espectacular en el periodo analizado (más de 15 puntos porcentuales). Aunque 
con excepciones, puede observarse cierto debilitamiento de los lazos con las economías maduras de la UE (a resaltar el retroceso de Francia) y una mayor conexión con economías de Europa del Este como Polonia. En el caso de Portugal, el carácter regional de su participación en CGV es aún más contundente que en el caso de España pues los diez principales destinos son europeos y suman el $85 \%$ del total.

\section{Conclusiones}

Desde la Gran Recesión, asistimos a un freno en el avance de las cadenas globales de valor. Los factores que guiaron la globalización de estas cadenas desde mediados de los noventa han dejado de operar en dicha dirección, contribuyendo a una reconfiguración en la que se intensifica su dimensión regional, lo que implica cierta vuelta a los orígenes de las cadenas transfronterizas de producción.

En el contexto de la Unión Europea, el componente regional de las cadenas transnacionales de valor siempre ha sido relevante, predominando prácticamente en todo el periodo de análisis, a pesar del intenso avance del componente global que solo se interrumpe en los años más recientes. Nuestro análisis ha puesto de manifiesto que a ello ha contribuido la activa participación de España y Portugal en las cadenas europeas de producción y su integración bilateral dentro de dichas cadenas.

Esta activa participación en CGV de España y Portugal ha crecido desde comienzos de siglo, al menos hasta 2011, tanto desde el punto de vista de su demanda de inputs intermedios, con una mayor dependencia importadora de sus exportaciones, como de la oferta, mediante un creciente peso de los inputs que suministran a los mercados exteriores para su transformación y exportación posterior. No obstante, se ralentiza y estanca en los años más recientes (2012-2014), si bien no llega a retroceder como ocurre en otras economías de nuestro entorno.

En cuanto a la dimensión geográfica de esta participación en cadenas transnacionales de producción, hemos observado una elevada persistencia del componente regional y, dentro de este, de la integración bilateral; así, existe una sólida conexión entre España y Portugal que se ha reforzado a lo largo del siglo, ya sea como origen del valor añadido foráneo contenido en las exportaciones del país o como destino del valor añadido doméstico que se incorpora en las exportaciones de terceros países. En cualquier caso, se trata de una integración bilateral asimétrica. Es mucho mayor la relevancia de España para Portugal, ya sea como suministrador de inputs a incorporar en las exportaciones lusas que como destino del valor añadido luso que se incorpora en las exportaciones de terceros países. Estas cadenas de producción ibéricas se integran, a su vez, en cadenas europeas, en las que Alemania, y en menor medida Francia (y de forma decreciente), ostentan un claro liderazgo y que han experimentado cierta reconfiguración hacia Europa del Este, lo que ha contribuido a que la dimensión regional siga siendo muy relevante. Unas redes de producción ibéricas o polo ibérico que se mantiene o incluso se hace más sólido en un contexto 
de reorganización geográfica de las CGV hacia China, dotándolas de una dimensión más global. Dentro de estas redes ibéricas, Portugal exhibe un sesgo hacia la participación backward, esto es, prima su papel como transformador de inputs intermedios foráneos procedentes de España destinados a la exportación, si bien se ha suavizado en el periodo de análisis. Sin embargo, en España prevalece en las redes con Portugal la participación forward, en tanto que es mayor su protagonismo como proveedor de inputs intermedios a la economía lusa desde donde se re-exportan a otros países.

Hemos cerrado este trabajo analizando la posición de España y Portugal en las cadenas de producción transnacionales del sector de vehículos de motor, dada su elevada propensión exportadora en ambas economías vinculada a las estrategias de fragmentación y deslocalización de la producción hacia la Península Ibérica por parte de las grandes empresas multinacionales del sector. La Península Ibérica presenta un notable grado de integración bilateral y con otros Estados miembros de la Unión Europea en el seno de las cadenas productivas automoción. Un polo ibérico que ha tendido a consolidarse al tiempo que han ido creándose lazos nuevos con economías de Europa del Este, ampliándose la dimensión de la red regional europea en la que ya participaban, y con China, permitiendo una integración en cadenas de ámbito global; de manera que ambas tendencias, regionalización y globalización de las cadenas transfronterizas en las que participan España y Portugal parecen haber coexistido al menos hasta mediados de la década que acabamos de cerrar.

\section{Referencias bibliográficas}

Amador, J., \& Stehrer, R. (2014). Portuguese exports in the Global Value Chains. Banco de Portugal Economic Bulletin, Abril, 64-78.

Antràs, P. (2020). Conceptual aspects of Global Value Chains (NBER Working Paper, No. 26539). https://www.nber.org/papers/w26539

Antràs, P., \& de Gortari, A. (2020). On the Geography of Global Value Chains. Econometrica, 88(4), 1553-1598.

Artuc, E., Bastos, P., \& Rijkers, B. (2018). Robots, Tasks and Trade. Policy Research Working Paper No. 8674. The World Bank.

Baldwin, R. (2011). Integration of the North American Economy and New-paradigm Globalization. En A. Sydor, Global Value Chains: Impacts and Implications (pp. 43-76). Foreign Affairs and International Trade Canada.

Baldwin, R., \& López-Gónzalez, J. (2015). Supply-chain Trade: A Portrait of Global Patterns and Several Testable Hypotheses. The World Economy, 38(11), 1682-1721.

Baldwin, R., \& Tomiura, E. (2020). Thinking ahead about the trade impact of COVID-19. En R. Baldwin \& B. W. Di Mauro, Economics in the Time of COVID-19 (pp. 59-70). A VoxEU.org eBook. 
Boffa, M., Jansen, M., \& Solleder, O. (2018). Value chain activity in the age of changing trade alliances. VOX, CEPR Policy Portal.

https://voxeu.org/article/value-chain-activity-age-changing-trade-alliances

Comisión Europea (2019). European Economic Forecast. Autumn 2019. Economic and Financial Affairs.

Constantinescu, C., Matoo, A., \& Ruta, M. (2015). The Global Trade Slowdown. Cyclical or Structural? Policy Research Working Paper No. 7158. The World Bank.

De Backer, K. \& Flaig, D. (2017). The Future of Global Value Chains. Business as usual or a "new normal"? OECD Publishing.

Díaz-Mora, C., Gandoy, R., \& Gónzalez-Díaz, B. (2018). Strengthening the Stability of exports through GVC Participation: Who, how and with whom matters. Journal of Economic Studies, 45(3), 603-637.

Gaulier, G., Santoni, G., Taglioni, D., \& Zignago, S. (2015). The power of the few in determining trade acceleratios and slowdowns. En B. Hoekman, The Global Trade Slowdown: A New Normal? (93-108). VoxEU eBook, CEPR Press y EUI.

Gaulier, G., Sztulman, A., \& Üzal, D. (2020). Are global value chains receding? The jury is still out. Key findings from the analysis of deflacted world trade in parts and components. International Economics, 161, 219-236.

Gordo, E., Kataryniuk, I., \& Santabárbara, D. (2020). El auge del proteccionismo: Implicaciones para la economía global. Economistas (166), 67-79.

Johnson, R. C., \& Noguera, G. (2017). A portrait of trade in value-added over four decades. Review of Economics and Statistics, 99(5), 896-911.

Kee, H. L., \& Tang, H. (2016). Domestic Value Added in Exports: Theory and Firm Evidence from China. American Economic Review, 106(6), 1402-36.

Koopman, R., Powers, W., Wang, Z., \& Wei, J. (2010). Give Credit Where Credit Is Due: Tracing Value Added in Global Production Chains (NBER Working Paper No. 16426). https://www.nber.org/papers/w16426

Koopman, R., Wang, Z., \& Wei, J. (2014). Tracing Value Added and Double Counting in Gross Exports. American Economic Review, 104(2), 459-94.

Los, B., Stherer, R., \& de Vries, G. (2015). Global value chains: "Factory World" is emerging. En J.Amador \& F. di Mauro, The Age of Global Value Chains (pp. 36-47). Voxeu. CEPR Press.

Marin, D. (3 de April de 2020). How COVID-19 Is Transforming Manufacturing. Project Syndicate. https://www.project-syndicate.org/columnist/dalia-marin

McKinsey (2019). Globalization in transition: the future of trade and global chains. McKinsey Global Institute.

Piatanesi, B., \& Arauzo-Carod, J. M. (2019). Backshoring and nearshoring: An overview. Growth and Change, 50(3), 806-823.

Strange, R., \& Zucchella, A. (2017). Industry 4.0, global value chains and international business. Multinational Business Review, 25(3), 174-184. https://doi.org/10.1108/MBR-052017-0028

Sturgeon, T. J., \& Van Biesebroeck, J. (2009). Crisis and Protection in the Automotive Industry. A Global Value Chain Perspective. Policy Research Working Paper No. 5060. The World Bank.

TheEconomist(15February 2020).Adeadly disease disrupts.https://www.economist.com/international/2020/02/15/the-new-coronavirus-could-have a-lasting-impact-on-global-supplychains. 
Timmer, M. P., Los, B., Stehrer, R., \& de Vries, G.J. (2016). An Anatomy of the Global trade Slowdown based on the WIOD 2016 Release. GGDC research memorandum number 162. World Bank (2020). Trade Development Report 2020: Trading for development in the age of Global Value Chains. https://www.worldbank.org/en/publication/wdr2020. 